\title{
A New Method for Handling Lockout Constraints on Controlled TCL Aggregations
}

\author{
Ziras, Charalampos; You, Shi; Bindner, Henrik W.; Vrettos, Evangelos
}

Published in:

Proceedings of 20th Power System Computation Conference

Link to article, DOI:

10.23919/PSCC.2018.8442907

Publication date:

2018

Document Version

Peer reviewed version

Link back to DTU Orbit

Citation (APA):

Ziras, C., You, S., Bindner, H. W., \& Vrettos, E. (2018). A New Method for Handling Lockout Constraints on Controlled TCL Aggregations. In Proceedings of 20th Power System Computation Conference IEEE. https://doi.org/10.23919/PSCC.2018.8442907

\section{General rights}

Copyright and moral rights for the publications made accessible in the public portal are retained by the authors and/or other copyright owners and it is a condition of accessing publications that users recognise and abide by the legal requirements associated with these rights.

- Users may download and print one copy of any publication from the public portal for the purpose of private study or research.

- You may not further distribute the material or use it for any profit-making activity or commercial gain

- You may freely distribute the URL identifying the publication in the public portal 


\section{A New Method for Handling Lockout Constraints on Controlled TCL Aggregations}

\author{
Charalampos Ziras, Shi You, Henrik W. Bindner \\ Department of Electrical Engineering \\ Technical University of Denmark \\ Roskilde, Denmark \\ \{chazi, sy, hwbi\}@elektro.dtu.dk
}

\author{
Evangelos Vrettos \\ Grid Integration Group \\ Lawrence Berkeley National Laboratory \\ Berkeley, California, USA \\ evrettos@lbl.gov
}

\begin{abstract}
Thermal loads are recognized as a valuable source of flexibility in face of the increasing variability caused by the large shares of renewable production. Lockout constraints can significantly reduce the flexibility of thermostatically controlled loads (TCLs). We propose a novel way of modifying the loads' lockout durations to achieve non-intrusive centralized control without relying on local computations and estimations. We derive analytical expressions for the flexibility reduction and validate them via simulations, which show that the proposed method describes the TCLs flexibility accurately. We further show that a simple stochastic centralized controller, which does not rely on local temperature measurements, outperforms the commonly used priority-stack controller in terms of system robustness against infeasible trajectories.
\end{abstract}

Index Terms-Aggregation, lockout constraints, stochastic controller, thermal battery model, thermostatically controlled loads.

\section{INTRODUCTION}

Due to the increasing shares of intermittent renewable energy production and the reduction of conventional generators capacity, it is expected that the power system's balancing needs will increase [1]. Buildings can be used for balancing services because they can act as virtual storage due to their thermal capacity. Using residential buildings flexibility in particular, has been found to be a viable investment [2]. Because of the large number of loads which must be controlled in order to provide a meaningful service, aggregation methods are necessary to represent the collective dynamics both for scheduling and control purposes. In [3] the potential of a collection of thermostatically controlled loads (TCLs) to arbitrage in the day-ahead energy market was investigated, by comparing two methods. In the first method the savings potential was calculated by optimizing the consumption of each TCL separately, whereas the second method used an aggregated thermal energy storage model, resulting in a linear optimization problem, which is computationally more attractive. It was shown that the aggregated model can achieve good performance in describing the population's dynamics.

Many research works have investigated how a collection of TCLs can be represented by a virtual battery model, which can describe its flexibility in a simple manner. Hao et al. proposed a stochastic battery model with varying energy capacity and power limits to describe the aggregate TCLs flexibility [4], [5].
An important contribution of this work is that the proposed battery model can be bounded by two other battery models, whose parameters are analytically calculated. Moreover, for homogeneous loads all models converge to one and therefore an exact battery model can be derived (exact means that any feasible trajectory provided by the battery model is also feasible for the individual loads). Another approach for describing flexibility is via polytopes, which is however much more demanding computationally [6]. A common limitation on the operation of TCLs is the so-called lockout constraints. This constraint forces a TCL to remain at the on or off state for a minimum duration after switching to protect it from wear. In [7] the effect of lockout constraints on the TCLs ramping capability was examined, whereas in [8]-[10] controllers were designed which take the lockout durations into account. However, their effect on the aggregation's energy and power limits was not addressed.

In this paper we propose a new battery model which accounts for the effect of lockout constraints and we quantify the impact on the energy and power limits of the population. We must note that we consider "large" time steps in the battery model, as in [3], [11]. Additionally, we show that if the lockout durations are not chosen appropriately, the TCLs might cycle outside their thermostat limits while being controlled, resulting in user-comfort violation. Our work is closer to [11], where the authors addressed the issue of reduced flexibility due to the lockout constraints. In contrast to our work which considers aggregate models, [11] modifies the power/energy limits of individual TCLs and relies on local intelligence to guarantee non-intrusive control. Moreover, the dependence of these limits on time-varying externalities such as the ambient temperature is neglected.

The main contribution of our work is a method to modify the lockout durations based on the expected baseline consumption and ensure the non-intrusive nature of the centralized control. We calculate the allowed combinations of lockout durations with which a TCL can always operate within its temperature deadband. The advantage of this approach is that there is no need for local computations for the loads to decide whether to follow the external control signal, which was a limitation in [11]. In a second contribution, we use the modified lockout durations to abstract the effects of lockout constraints and 
characterize the aggregation's flexibility in a more accurate manner. We have considered the effect of lockout constraints on aggregations of TCLs offering primary frequency control in our previous work [12], [13], but we didn't quantify the flexibility reduction in terms of power and energy. Finally, we show that a simple stochastic controller without local temperature measurements is more robust than the commonly used priority-stack controller, which results in large oscillations in case of intractable reference set-points.

The remainder of the paper is organized as follows. In Section II the basic principles of TCL modeling and the thermal battery model are introduced. In Section III a method to modify the lockout constraints is presented, as well as analytical expressions of their effect on the energy and power limits of the battery model. Section IV discusses and compares two different control approaches, namely the stochastic and priority-stack controller. In Section $\mathrm{V}$ simulation results are presented and Section VI concludes.

\section{MODELING OF TCLS}

\section{A. Individual TCL modeling}

We use a first-order model to describe the evolution of a TCL's air temperature. Higher order models exist in the literature, for instance the works of [9], [8], where the building's mass temperature is also considered. However, similar to other works, we believe that this model can sufficiently capture the TCLs dynamics for our purposes [14], [3]. Consider the case of a heating TCL $i$ which is controlled by an on/off hysteresis controller. The following first-order differential equation describes the evolution of the lumped temperature $T_{i}(t)$ of the the air and building mass

$$
\begin{array}{ll}
\dot{T}_{i}(t)=\alpha_{i}\left[T_{\mathrm{a}, i}(t)-T_{i}(t)\right]+w_{i}(t) & \text { when OFF } \\
\dot{T}_{i}(t)=\alpha_{i}\left[T_{\mathrm{a}, i}(t)-T_{i}(t)\right]+\beta_{i} P_{\mathrm{n}, i}+w_{i}(t) & \text { when ON, }
\end{array}
$$

where $\alpha=1 / R C$, and $\beta=\eta / C ; C$ is the thermal capacitance, $R$ is the thermal resistance, $T_{\mathrm{a}}(t)$ is the time-varying outside temperature, $\eta$ is the coefficient of performance $(\mathrm{COP})^{1}$, and $P_{\mathrm{n}}$ is the nominal compressor power. Term $w(t)$ can be considered a Gaussian noise term with zero mean and small variance like in [7], or it can aggregate the effect of the system disturbances, such as door and window openings, occupancy etc., similar to [14]. Denote by $T_{\mathrm{r}, i}$ the set-point of the TCL and by $\Delta_{i}$ its deadband; the hysteresis controller will switch the compressor on/off in order to keep the temperature within the temperature region $\left[T_{\mathrm{r}, i}-\Delta_{i}, T_{\mathrm{r}, i}+\Delta_{i}\right]$. By dropping the index $i$ for convenience, the temperature evolution can be described in discrete time by

$$
T_{t+1}=a T_{t}+b m_{t}+c\left(T_{\mathrm{a}, t}+w_{t}\right)
$$

where $a=e^{-\Delta t /(R C)}, b=(1-a) \eta R P_{\mathrm{n}}, c=(1-a), \Delta t$ is the model's discretization time step, and $m_{t} \in\{0,1\}$ is the on/off state. Using the discrete time model, and by setting

\footnotetext{
${ }^{1}$ In our analysis we considered a constant COP for simplicity, but a varying COP can be used in the model as a parameter, similar to $T_{\mathrm{a}}$.
}

$Q=\eta R P_{\mathrm{n}}$ for notation simplicity, we can derive the duration of the on $\left(t^{\text {on }}\right)$ and off cycles $\left(t^{\text {off }}\right)$

$$
\begin{aligned}
& t_{i}^{\text {on }}=R_{i} C_{i} \ln \left[\frac{T_{r, i}-\Delta_{i}-T_{\mathrm{a}, i}-Q_{i}}{T_{r, i}+\Delta_{i}-T_{\mathrm{a}, i}-Q_{i}}\right], \\
& t_{i}^{\text {off }}=R_{i} C_{i} \ln \left[\frac{T_{r, i}+\Delta_{i}-T_{\mathrm{a}, i}}{T_{r, i}-\Delta_{i}-T_{\mathrm{a}, i}}\right] .
\end{aligned}
$$

The device's duty cycle is equal to $D_{i}=t_{i}^{\text {on }} /\left(t_{i}^{\text {on }}+t_{i}^{\text {off }}\right)$, whereas the average consumption is equal to $P_{\mathrm{av}, i}=P_{\mathrm{n}, i} D_{i}$. We must note here that external temperature $T_{\mathrm{a}}$ is considered common for all the loads. Furthermore, the time-varying nature of $T_{\mathrm{a}}$ and the external disturbances result in time-varying duty cycles. Modeling the aggregate effect of disturbances such as the door/window openings requires statistical approaches due to their event-based nature and is outside the scope of the paper. We use however a time-varying $T_{\mathrm{a}}$ to show the effect of a non-constant duty cycle on the aggregate flexibility.

\section{B. Continuous-power model}

The aggregated consumption of $n$ loads is given by the summation of their individual states, multiplied by their nominal power consumption. The nonlinearity of the presented hybrid model makes it inconvenient for analysis and optimization. A more suitable model for relatively large populations of TCLs is the continuous-power model, where a continuous state $u_{i}(t) \in\left[0, P_{\mathrm{n}, i}\right]$ is used for the power consumption, instead of a binary variable. The equivalence of the deadband and continuous-power models has been discussed in [5], [7] and was shown to be a good approximation for a relatively small heterogeneity and a sufficient number of loads. As a result, temperature is given by

$$
\dot{T}_{i}(t)=-\alpha_{i}\left[T_{i}(t)-T_{\mathrm{a}, i}(t)\right]+\beta_{i} u_{i}(t)+w_{i}(t) .
$$

In this case the temperature must be maintained within the desired boundaries by controlling the continuous power input.

\section{Thermal battery model}

Thermal battery models (TBMs) have been proposed and investigated in many works as a convenient representation of the flexibility of a population of TCLs for scheduling and control purposes [15], [5], [3]. We follow an approach similar to [5], [3] and we express the aggregated flexibility of a pool of TCLs with relatively low heterogeneity via a TBM considering the average parameter values. In that case the baseline consumption of $n$ TCLs, i.e. the load that maintains the aggregation's average temperature at the set-point value, is given by

$$
P_{k}^{\mathrm{b}}=\frac{n \bar{\alpha}\left(T_{\mathrm{r}}-T_{\mathrm{a}, k}\right)}{\bar{\beta}},
$$

where the upper bar indicates the average value and index $k$ indicates the battery model's time step, whose duration is equal to $t_{\mathrm{BM}}$; this duration is not to be confused with the hybrid model's time step $\Delta t$ used in the simulations. State $X_{k}$ represents the state of charge of the battery model and its evolution is described by

$$
X_{k+1}=X_{k} a_{\mathrm{d}}+\left(P_{k}-P_{k}^{\mathrm{b}}\right) t_{\mathrm{BM}},
$$


TABLE I: TCLs Parameters

\begin{tabular}{c|c|c}
\hline Parameter & Description & Value \\
\hline$T_{\mathrm{r}}\left({ }^{\circ} \mathrm{C}\right)$ & Temperature set-point & 22.5 \\
$C\left(\mathrm{kWh} /{ }^{\circ} \mathrm{C}\right)$ & Thermal capacitance & 2 \\
$R\left({ }^{\circ} \mathrm{C} / \mathrm{kW}\right)$ & Thermal resistance & 2 \\
$P_{\mathrm{n}}(\mathrm{kW})$ & Nominal power & 5.6 \\
$\eta(-)$ & Coefficient of performance & 3 \\
$\Delta\left({ }^{\circ} \mathrm{C}\right)$ & Temperature deadband & 0.5 \\
\hline
\end{tabular}

where the self-discharge rate $a_{\mathrm{d}}$ is equal to $1-\bar{\alpha} t_{\mathrm{BM}} . P_{k}$ is the total consumption and can take values in the range $\left[0, n \bar{P}_{\mathrm{n}}\right]$. State $X$ is limited by the capacity $S$ of the battery model, which is expressed in $\mathrm{kWh}$, and is calculated as

$$
S=\frac{n \bar{\Delta}}{\bar{\beta}} .
$$

In this paper we consider TCL flexibility via a TBM over "large" steps, similar to [3], and thus the reference is constant over a larger period and does not change every few seconds, like in TBMs used for frequency regulation, such as [15], [5]. However, several works have shown that the effective power range is smaller than $\left[0, n \bar{P}_{\mathrm{n}}\right]$. Moreover, the presence of lockout constraints further reduces the loads flexibility, whereas a local controller is required to evaluate if an external control signal will result in thermostat limits violations, further complicating the analytical characterization of flexibility. In the following section we will present a method for modifying the lockout durations to guarantee user comfort and we will calculate the energy and power limits of the TBM of TCLs under lockout constraints. By modifying these durations we can capture the effects of these constraints on the aggregate flexibility, abstract their behaviour close to the thermostat limits and derive analytical forms for the new energy and power limits of the TBM. For our study we consider a population of TCLs with relatively small heterogeneity (10\%). Our simulations showed that the proposed approach works well even with higher heterogeneity levels, but it is common to cluster the loads in the case of large heterogeneity to achieve better performance. The parameters used in our simulations are shown in Table I and are taken from [16].

\section{EXTENDED BATTERY MODEL WITH LOCKOUT CONSTRAINTS}

\section{A. Modification of the lockout durations}

A common practical constraint on the operation of TCLs is a minimum lock-on duration $t_{\mathrm{on}, i}^{1}$, which forces the TCL to remain at the on state once switched on. Similarly, a minimum lock-off duration $t_{\text {off }, i}^{1}$ locks the TCL at the off state. As a result of these constraints, if the TCL's duty cycle is not equal to 0.5 , then a switching action can force it to operate outside its temperature deadband if the lockout durations are not carefully chosen. To illustrate this behaviour consider a TCL with the parameters presented in Table I. We assume that both durations are set by the manufacturer equal to 3 minutes. We consider a case where $T_{\mathrm{a}}=10^{\circ} \mathrm{C}$ and $D=37 \%$ and a case where $T_{\mathrm{a}}=0^{\circ} \mathrm{C}$ and $D=67 \%$. The temperature evolutions when the TCL continuously receives a command to switch on and off are shown in Fig. 1.

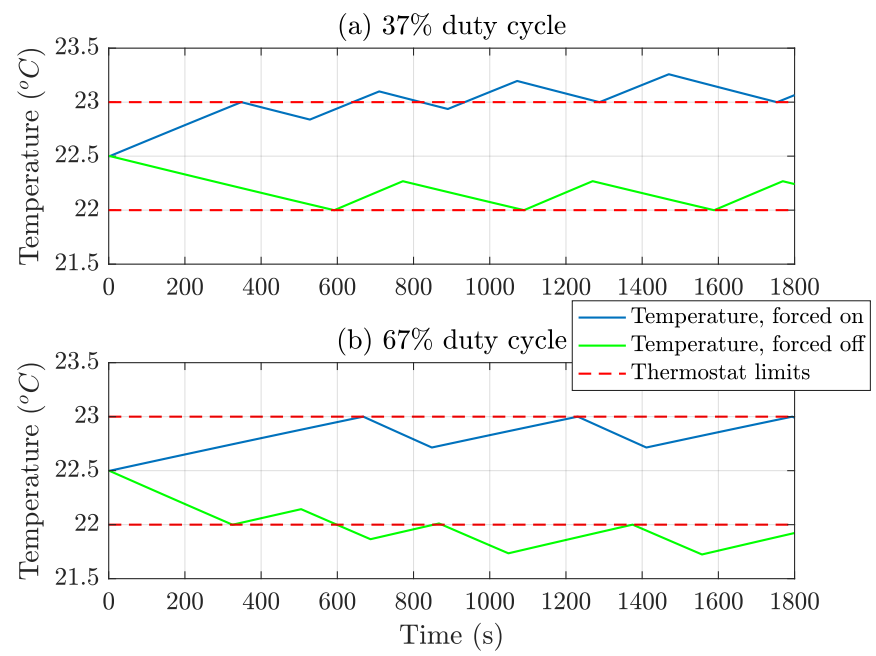

Figure 1: Violation of thermostat limits due to equal lockout durations.

When $D<50 \%$ then $t_{\text {on }}<t_{\text {off }}$ and the heating rate is larger than the cooling rate. If the TCL is forced to switch on, then once it reaches the upper thermostat limit it will switch

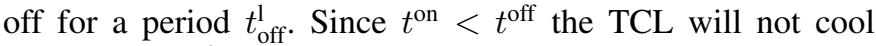
enough after $t_{\text {off }}^{1}$ has elapsed and a switching will force it to remain on for $t_{\mathrm{on}}^{1}$, thus exceeding the thermostat limit. This however will not occur when the load is forced to remain off, since it is able to increase the temperature enough during the $t_{\text {off }}^{1}$ period that it operates after reaching the lower thermostat limit. Following similar arguments, it is expected that the load will only exceed the lower thermostat limit if forced to switch off when $D>50 \%$, as seen in Fig. 1. To prevent the TCL from operating outside the deadband, the local controller must disregard external signals which will force the TCL in such regions. However, it is difficult to abstract the effect of such temperature-dependent local operations on an aggregate level and characterize the aggregated behaviour of the population. For this reason we propose to modify the individual lockout durations. We have presented this concept in our earlier work for individual TCLs [12], [13] and here we formalize and extend this concept, in order to incorporate it to a TBM.

Consider the case where a TCL is forced to remain on and switches off for $t_{\text {off }}^{1}$ after reaching the upper limit. Once the TCL switches on, a maximum lock-on duration is required, such that temperature does not exceed this limit. For notation simplicity we drop the subscript $i$ and denote by $T_{\min }$ and $T_{\max }$ the lower and upper thermostat limit respectively. By using (2) it is straightforward to show that the following inequality must hold, in order to avoid temperatures higher than $T_{\max }$

$$
t_{\text {on }}^{1} \leq R C \ln \left[\frac{\left(T_{\max }-T_{\mathrm{a}}\right) e^{\frac{-t_{\mathrm{off}}^{1}}{R C}}-Q}{T_{\max }-T_{\mathrm{a}}-Q}\right] .
$$

For our example with $T_{\mathrm{a}}=10^{\circ} \mathrm{C}$ and $t_{\text {off }}^{1}=180 \mathrm{~s}$, the maximum allowed $t_{\mathrm{on}}^{1}$ is approximately $113 \mathrm{~s}$. An upper bound is thus required for the lock-on duration, so that it is 
small enough to prohibit the TCL from operating in higher temperatures. When the load is forced to remain off, a lower limit on $t_{\text {on }}^{1}$ must be set, such that temperature increases enough before the load switches off. To avoid temperatures lower than $T_{\min }$, the following inequality must hold

$$
t_{\mathrm{on}}^{1} \geq R C \ln \left[\frac{\left(T_{\min }-T_{\mathrm{a}}-Q\right) e^{\frac{-t_{\mathrm{off}}^{1}}{R C}}}{T_{\min }-T_{\mathrm{a}}-Q e^{\frac{-t_{\text {off }}^{1}}{R C}}}\right] .
$$

In our example (10) results in a minimum lock-on duration of 101 s. A lower bound is thus imposed on the lock-on duration, which will increase the temperature enough, so that a lock-off duration of $180 \mathrm{~s}$ will not result in temperatures lower than $T_{\min }$. Inequalities (9) and (10) define feasible regions for the lockout durations, which guarantee non-violation of both thermostat limits for any external signal. Additional constraints may be imposed by the minimum lockout durations set by the manufacturer. The allowed combinations for $t_{\text {off }}^{1}$ and $t_{\mathrm{on}}^{1}$ are shown in Fig. 2 for 3 duty cycles: larger than $50 \%$, smaller than $50 \%$, and equal to $50 \%$.

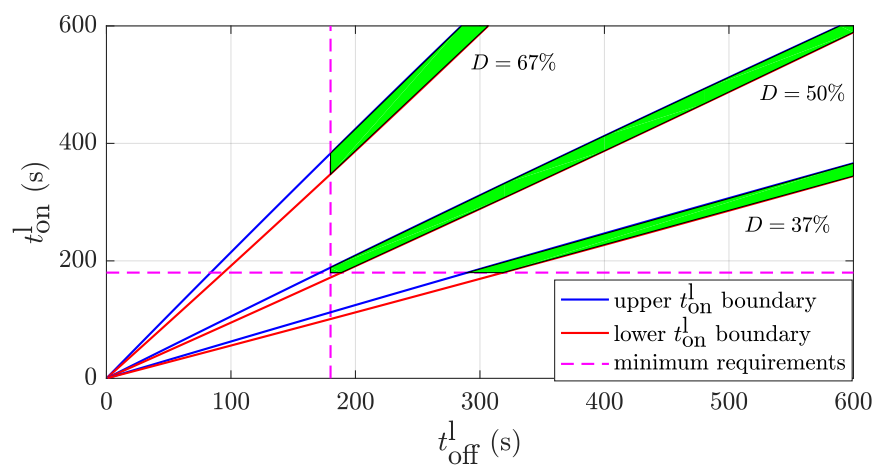

Figure 2: Allowed region (area with green color) for $t_{\mathrm{off}}^{1}$ and $t_{\mathrm{on}}^{1}$.

For combinations of $t_{\text {off }}^{1}$ and $t_{\mathrm{on}}^{1}$ within the allowed regions, user comfort is always guaranteed. Interesting results can be drawn by observing Fig. 2 . First, the ratio $t_{\text {on }}^{1} / t_{\text {off }}^{1}$ must be almost equal to 1 for $D=50 \%$, whereas it must increase as $D$ increases and decrease as $D$ decreases. Second, if one of the durations is fixed at its minimum value, an allowed range for the other duration exists. The closer the lockout value is to the boundaries, the more sensitive the TCL becomes to changes of the external temperature, as we will show next.

Consider the case where $D=37 \%$ and $T_{\mathrm{a}}=10^{\circ} \mathrm{C}$, then for $t_{\mathrm{on}}^{1}=180 \mathrm{~s}, t_{\mathrm{off}}^{1} \in[290,318] \mathrm{s}$. The lower limit $t_{\text {off }}^{1, \min }$ represents the smallest duration which decreases the temperature enough, so that an immediate switching after $t_{\text {off }}^{1, \text { min }}$ will not result in a temperature higher than $T_{\max }$. A realized $T_{\mathrm{a}}$ lower than $10^{\circ} \mathrm{C}$ will increase the cooling rate and lead the TCL to an even lower temperature, thus avoiding a deadband violation. However, a higher realized $T_{\mathrm{a}}$ will have the opposite effect, as it will slow down the TCL's cooling rate. By setting $t_{\text {off }}^{1}$ equal to $t_{\text {off }}^{1, \text { min }}$, any temperature higher than $10^{\circ} \mathrm{C}$ will violate the upper thermostat limit. By increasing the value of $t_{\text {off }}^{1}$ the TCL is able to remain within the deadband for larger realized $T_{\mathrm{a}}$ values, providing some robustness against $T_{\mathrm{a}}$ variations.
The relationship between $T_{\mathrm{a}}$ and $t_{\text {off }}^{1}$ is given by solving (9) using a fixed $t_{\mathrm{on}}^{\mathrm{l}}$ (in our case equal to $180 \mathrm{~s}$ ), resulting in

$$
T_{\mathrm{a}}^{\mathrm{up}}\left(t_{\mathrm{off}}^{1}\right)=\frac{T_{\max }\left(e^{\frac{-t_{\mathrm{off}}^{1}}{R C}}-e^{\frac{t_{\mathrm{on}}^{1}}{R C}}\right)+Q\left(e^{\frac{t_{\mathrm{on}}^{1}}{R C}}-1\right)}{e^{\frac{-t_{\mathrm{off}}^{1}}{R C}}-e^{\frac{t_{\mathrm{on}}^{1}}{R C}}} .
$$

Following similar arguments, by setting $t_{\text {off }}^{1}$ equal to $t_{\text {off }}^{1, \max }$, any $T_{\mathrm{a}}$ lower than $10^{\circ} \mathrm{C}$ will result in a $T_{\text {min }}$ limit violation. By increasing $t_{\text {off }}^{1}$, the TCL is able to remain within the deadband for smaller realized $T_{\mathrm{a}}$ values. The relationship between $T_{\mathrm{a}}$ and $t_{\text {off }}^{1}$ is given by solving (10), resulting in

$$
T_{\mathrm{a}}^{\mathrm{d}}\left(t_{\mathrm{off}}^{1}\right)=\frac{T_{\min }\left(e^{\frac{-t_{\mathrm{off}}^{1}}{R C}}-e^{\frac{t_{\mathrm{on}}^{1}}{R C}}\right)+Q e^{\frac{-t_{\text {off }}^{1}}{R C}}\left(e^{\frac{t_{\mathrm{on}}^{1}}{R C}}-1\right)}{e^{\frac{-t_{\mathrm{off}}^{1}}{R C}}-e^{\frac{t_{\mathrm{on}}^{1}}{R C}}} .
$$

It is evident that there is a trade-off in the robustness against $T_{\mathrm{a}}$ when choosing $t_{\text {off }}^{1}$. For smaller values, the TCL has a relatively large margin against reduced $T_{\mathrm{a}}$ values, but a very small margin against $T_{\mathrm{a}}$ values larger than the expected. As $t_{\text {off }}^{1}$ increases, these margins vary, as seen in Fig. 3.

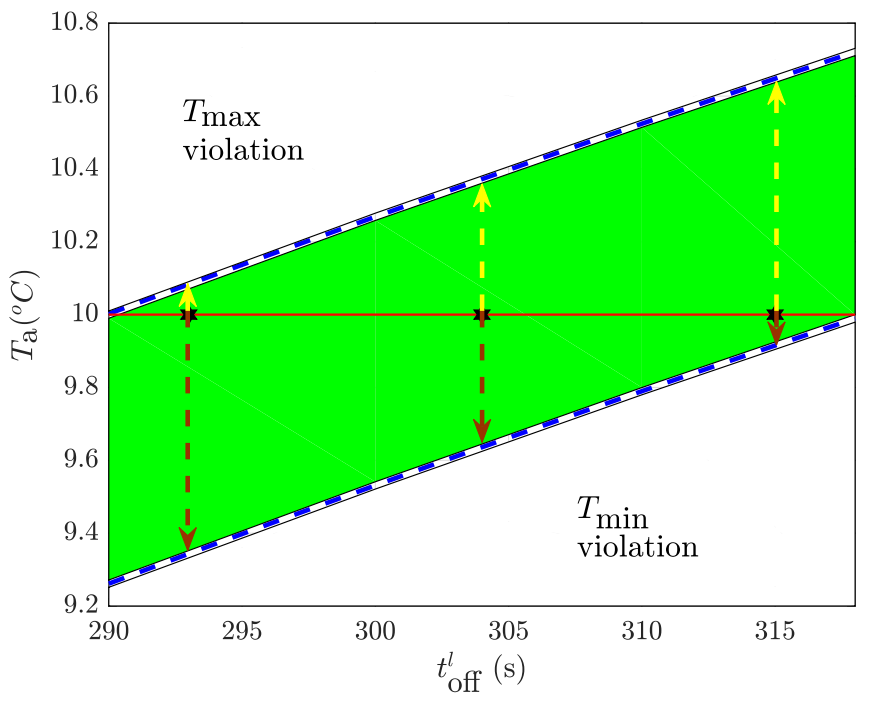

Figure 3: Robustness against $T_{\mathrm{a}}$ variation. The green area indicates the $T_{\mathrm{a}}$ values for which the TCL remains within the deadband for a set $t_{\text {on }}^{1}$ and varying $t_{\text {off }}^{1}$ values. The yellow arrows indicate robustness against $T_{\mathrm{a}}$ increase and the brown against $T_{\mathrm{a}}$ decrease.

It is reasonable to choose a $t_{\text {off }}^{1}$ value close to the middle of the allowed range, so that the TCL will not violate the thermostat limits with small $T_{\mathrm{a}}$ variations in either direction. For the considered example, by setting $t_{\text {off }}^{1}=304 \mathrm{~s}$, the TCL is robust against $T_{\mathrm{a}}$ for $T_{\mathrm{a}} \in[9.6,10.4]^{\circ} \mathrm{C}$. In practice, even if lockout durations are chosen according to the proposed method, there is a number of factors which can lead the TCL outside its deadband, such as disturbances (e.g. window openings and human interaction) and noisy measurements [17]. We consider only $T_{\mathrm{a}}$ variations, as an example of assessing the impact of uncertainty in choosing the lockout durations. However, by setting the appropriate ratio between the lockout durations, it is expected that if deadband violations occur, they will be small. 
Without exchanging internal temperature information with the aggregator, the absence of a local controller which evaluates the external control signals can force the load to cycle outside its deadband, as showcased in Fig. 1. A simple way to avoid this would be to temporarily increase the lockout duration if temperature crosses the thermostat limits but the TCL is still locked. This can be achieved without using any temperature measurements, i.e by counting time since the locked TCL crossed the thermostat limit and by temporarily modifying the lockout duration by using the appropriate ratio.

\section{B. Modification of energy capacity limits}

Once the required lockout durations have been chosen, their effect on the aggregation's performance can be investigated. Consider a TCL which is forced to remain on and as close as possible to its upper temperature limit. Once it reaches this limit, it will switch off and a period equal to $t_{\text {off }}^{1}$ will have to pass until it can switch on again. Due to the modified lockout durations, the TCL cycles within a temperature region $\Delta T^{+}$, below the $T_{\max }$, which can be calculated as

$$
\Delta T^{+}=\left(T_{\max }-T_{\mathrm{a}}\right)\left(1-e^{\frac{-t_{\mathrm{off}}^{1}}{R C}}\right) .
$$

Similarly, the TCL would be confined within a temperature region $\Delta T^{-}$close to $T_{\min }$, if forced to remain off, given by

$$
\Delta T^{-}=\left(1-e^{\frac{-t_{\mathrm{on}}^{1}}{R C}}\right)\left(T_{\mathrm{a}}+Q-T_{\min }\right) .
$$

A consequence of this behaviour is a reduced effective temperature deadband, imposed by the lockout constraints; instead of an allowed temperature band $\Delta$ above the set-point, the actual deadband is equal to $\Delta-0.5 \Delta T^{+}$. Also, the allowed deadband below the set-point is equal to $\Delta-0.5 \Delta T^{-}$. These modified deadbands can be interpreted as reduced $T_{\max }$ and increased $T_{\min }$ thermostat limits of the continuous-power model and are time-varying, since $T_{\mathrm{a}}$ and the lockout durations are not constant. Thus, the modified, time-varying capacity limits of the TBM can be differentiated between a positive $S_{k}^{+}$and a negative $S_{k}^{-}$, and can be calculated as

$$
S_{k}^{+}=\frac{n\left(\bar{\Delta}-0.5 \overline{\Delta T}_{k}^{+}\right)}{\bar{\beta}}, \quad S_{k}^{-}=\frac{n\left(\bar{\Delta}-0.5 \overline{\Delta T}_{k}^{-}\right)}{\bar{\beta}},
$$

where $\overline{\Delta T}^{+}$and $\overline{\Delta T}^{-}$can be calculated from (13) and (14) respectively, by using the population's average values.

\section{Modification of power limits}

Similar to energy capacity, the power limits of the aggregation, which we denote by $P_{k}^{-}$and $P_{k}^{+}$for the lower and upper limit respectively, are affected by the lockout constraints. Using (7) and (15) may result in $P_{k}$ values which cannot be tracked by the TCLs. As already stated, we describe flexibility on large steps, since our focus is not on the population dynamics when they track fast-changing reference signals. In general, imposing power limits on a TBM under lockout constraints for an arbitrary reference signal is very challenging, as also recognized in [7]. For relatively small deadband values, as is the case in most works in the literature, we found that the implicit power constraints imposed by (7), (15) result in power values which are generally tractable. One important assumption though is that $t_{\mathrm{BM}}$ is considerably longer than the lockout durations. We introduce the switching on $\left(s_{k}^{\text {on }}\right)$ and off $\left(s_{k}^{\text {off }}\right)$ rates, which express the load change in $\mathrm{kW} / \mathrm{s}$ by loads switching on and off respectively. These rates are approximately constant at steady state and are given by

$$
s_{k}^{\text {off }} \approx P_{k}^{\mathrm{b}} / \bar{t}_{k}^{\text {on }}, \quad s_{k}^{\text {on }} \approx\left(n \bar{P}_{\mathrm{n}}-P_{k}^{\mathrm{b}}\right) / \bar{t}_{k}^{\text {off }},
$$

where $\bar{t}_{k}^{\text {off }}$ and $\bar{t}_{k}^{\text {on }}$ are the average off and on times of the TCLs at period $k$. In steady state there is always a share of loads which is locked due to natural cycling. The steady state parts of the loads locked off $\left(L_{k}^{\mathrm{OFF}}\right)$ and on $\left(L_{k}^{\mathrm{OFF}}\right)$, expressed in $\mathrm{kW}$, are calculated by

$$
\begin{gathered}
L_{k}^{\mathrm{OFF}}=n \bar{P}_{\mathrm{n}} \cdot \bar{t}_{\mathrm{off}, k}^{1} /\left(\bar{t}_{k}^{\mathrm{on}}+\bar{t}_{k}^{\mathrm{off}}\right), \\
L_{k}^{\mathrm{ON}}=n \bar{P}_{\mathrm{n}} \cdot \bar{t}_{\mathrm{on}, k}^{1} /\left(\bar{t}_{k}^{\mathrm{on}}+\bar{t}_{k}^{\text {off }}\right),
\end{gathered}
$$

where $\vec{t}_{\text {off }, k}$ and $\vec{t}_{\text {on }, k}$ are the average lock-off and lock-on durations respectively. We refer the interested reader to our previous work [13] for more details on how to derive these approximations. A limitation on total power is imposed by the number of locked devices. Thus, starting from steady state, it's impossible to reduce the total load to zero because a load $L_{k}^{\mathrm{ON}}$ is not available to switch off. Following similar arguments it's not possible to increase the total load up to the installed capacity of the aggregation. These limits are expressed by

$$
P_{k}^{+}=n \bar{P}_{\mathrm{n}}-L_{k}^{\mathrm{OFF}}, \quad P_{k}^{-}=L_{k}^{\mathrm{ON}} .
$$

However, the number of locked devices changes when a $P_{k}$ other than $P_{k}^{\mathrm{b}}$ is tracked. Deriving analytical expressions to reflect this is very complicated. We can however impose stricter limits than (19). Consider the case where the system is in steady state, i.e. $X_{k}=0$ and $P_{k}=P_{k}^{\mathrm{b}}$, and a $P_{k+1} \geq P_{k}$ is calculated via (7), (15). We want to calculate the maximum allowed value of $P_{k+1}$ for a given $t_{\mathrm{BM}}$. We consider a prioritystack controller to describe the dynamics of the population. In the beginning, the required loads (equal to $P_{k+1}-P_{k}$ ) at the off state with the lowest temperatures will switch on. Let us denote by $t_{r 1}$ the average time period that the newly switchedon loads will need to reach the upper temperature limit and start switching off. Until $t_{\mathrm{r} 1}$, loads will continue to switch off at a rate $s_{k}^{\text {off }}$, equal to the pre-disturbance level, since the newly switched on loads were placed on the lower end of the temperature range and do not change the switching rate. Thus the pool of switched off and unlocked loads will decrease by $P_{k+1}-P_{k}$ but will remain constant until $t_{\mathrm{r} 1}$. After $t_{\mathrm{r} 1}$, there will be a continuous reduction of the available to switch on load due to the larger switching off rate due to the arrival of the initially switched on loads to the upper thermostat limit. The aggregation is unable to track the reference signal when all the available off load has switched on. Time periods $t_{\mathrm{r} 1}$ and after $t_{\mathrm{r} 2}$ are given by

$$
t_{\mathrm{r} 1}=\bar{t}_{k+1}^{\mathrm{on}} \cdot \frac{n \bar{P}_{\mathrm{n}}-P_{k+1}}{n \bar{P}_{\mathrm{n}}-P_{k}}, \quad t_{\mathrm{r} 2}=\frac{n \bar{P}_{\mathrm{n}}-P_{k+1}-L_{k+1}^{\mathrm{OFF}}}{P_{k+1} / \bar{t}_{k+1}^{\mathrm{on}}} .
$$


For a given $t_{\mathrm{BM}}$, the maximum $P_{k+1}$ value can be obtained by solving the equation: $t_{\mathrm{BM}}=t_{\mathrm{r} 1}+t_{\mathrm{r} 2}$. Following similar arguments, $t_{\mathrm{r} 3}$ and $t_{\mathrm{r} 4}$ can be calculated to find the minimum tractable $P_{k+1}$ and their values are given by

$$
t_{\mathrm{r} 3}=\bar{t}_{k+1}^{\mathrm{off}} \cdot \frac{P_{k+1}}{P_{k}}, \quad t_{\mathrm{r} 4}=\frac{P_{k+1}-L_{k+1}^{\mathrm{ON}}}{\left(n \bar{P}_{\mathrm{n}}-P_{k+1}\right) / \bar{t}_{k+1}^{\mathrm{off}}} .
$$

\section{CONTROL}

Various control approaches exist in the literature, with varying levels of complexity. A number of factors may have a significant effect on the control type and architecture, namely knowledge of the system's parameters, and the specific application, as well as the shared information and type of communication. In [18] a state-bin transition model was proposed, which models the temperature evolution of the TCLs as a Markov process. The look-ahead controller estimates the state of the system at the next control step and switches a number of TCLs on or off to achieve the best possible tracking accuracy. The controller either dispatches switching probabilities uniformly among the temperature bins or prioritizes the TCLs closer to their thermostat limits to avoid short cycling.

The latter approach, called priority stack controller, is often employed as a simple, centralized control method which minimizes switching and is usually employed as the most effective control strategy to minimize the switching actions and extract the maximum flexibility. A central controller receives the power consumption and temperature values of all units, calculates how many loads need to switch to track the reference signal and sends commands to the loads which are closest to switching. A major disadvantage of this approach is that internal temperatures are required at each control step, which increases the communication and sensing requirements; most importantly, the required resolution and accuracy for such a control scheme are much higher than what is practically feasible. Thus, some authors have proposed to employ a stochastic controller [10], [8], which after receiving the aggregated load value, dispatches a switching probability to the loads, which based on an individual random number drawn from a uniform distribution, decide to change their state or not.

In this work we examine both controllers and we show that a stochastic controller not only requires less information but is more robust than the priority-stack controller when infeasible trajectories are to be tracked. Even though both controllers have been proposed and used in the literature, the side-effects of using a priority-stack controller in the presence of lockout constraints have not been pointed out yet.

\section{RESUlts}

In this section we validate the energy and power limits of the proposed extended battery model (EBM) and we compare the performance of a stochastic and a priority-stack controller. We consider a population of 10000 TCLs with the parameters shown in Table I, a heterogeneity of $10 \%$, a simulation time step equal to $1 \mathrm{~s}$ and a constant $T_{\mathrm{a}}=5^{\circ} \mathrm{C}$. A stochastic controller is applied to track the reference signal in the first simulation. We set a power reference such that the upper and lower limits of the proposed EBM, as calculated by (15), are reached; we use the limits of the standard battery model (SBM), which neglects the lockout durations, to show the effect on flexibility. As can be seen in Fig. 4, the proposed model is able to capture the reduced energy limits of the aggregation with very high accuracy. At $t=2000 \mathrm{~s}$ all positive flexibility, i.e. the ability to track references higher than the baseline, is depleted and if the higher SBM energy limits were considered, then significant tracking errors would occur. In

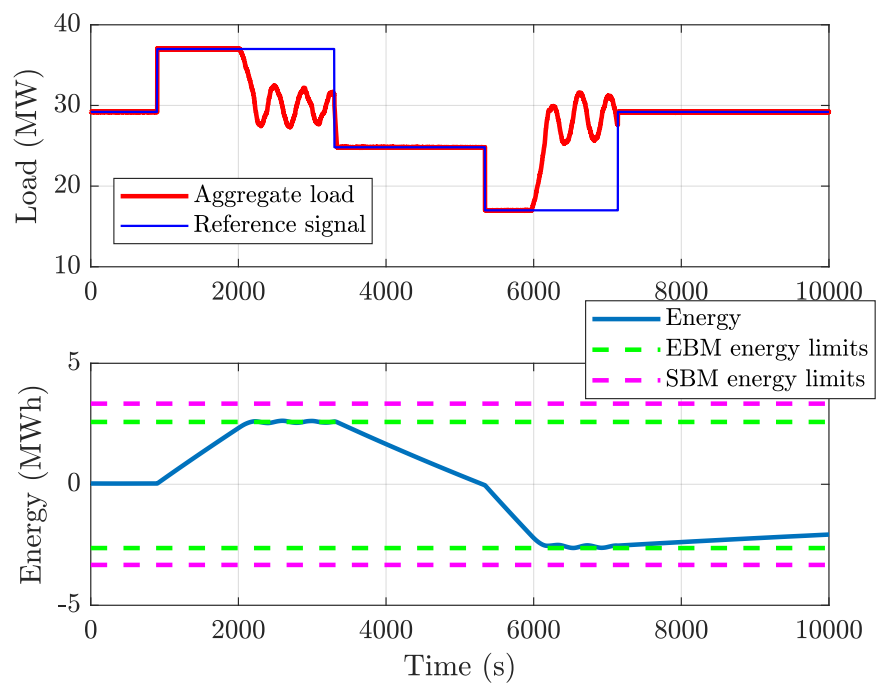

Figure 4: Validation of the energy limits of the proposed model.

the second simulation we apply a power reference signal to identify the power limits. This time we employed a prioritystack controller, since we used this type of controller to derive the power limits. We set $t_{\mathrm{BM}}=600 \mathrm{~s}$ and try to find the maximum power that can be tracked for this duration. Using (7), (15) we obtain a maximum load of 44.5 MW. As seen in Fig. 5 (a), the aggregation is not able to track the reference signal given by (7), (15) due to the power constraints. The theoretically calculated time when the reference signal could not be tracked, calculated via (20), was found to be almost the same as the simulated. By using $t_{\mathrm{BM}}=t_{\mathrm{r} 1}+t_{\mathrm{r} 2}$ and (20) the maximum tractable power was calculated as $42.8 \mathrm{MW}$, which as shown in Fig. 5 (b) can be tracked for the whole $t_{\mathrm{BM}}$ period.

We further evaluated the tracking performance and robustness of a stochastic and a priority-stack controller in the case of infeasible reference signals, as shown in Fig. 6. No significant reduction in flexibility was observed by using a simple stochastic controller which does not rely on internal temperature measurements. On the other hand, the priority-stack controller tends to synchronize the loads. This results in large oscillations in the aggregated load, once the reference signal cannot be followed. Notice that with the stochastic controller the loads oscillate around their baseline consumption with a period equal to $\vec{t}_{\mathrm{on}, k}+\vec{t}_{\mathrm{off}, k}$ and a relatively small amplitude; the other controller results in considerably larger load oscillations. Additionally, it forces the available on and off load to oscillate due to the induced synchronization, 
(a) Intractable power reference of $44.5 \mathrm{MW}$

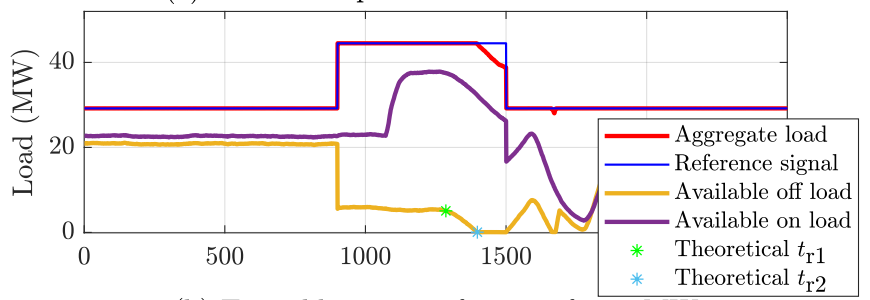

(b) Tractable power reference of $42.8 \mathrm{MW}$

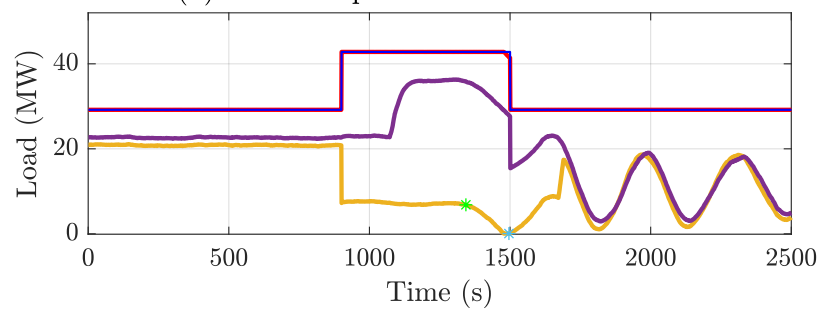

Figure 5: Validation of the power limits of the proposed EBM.

(a) Stochastic controller

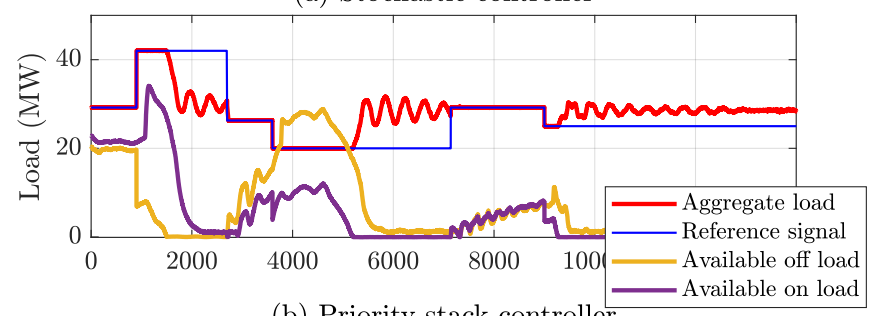

(b) Priority-stack controller

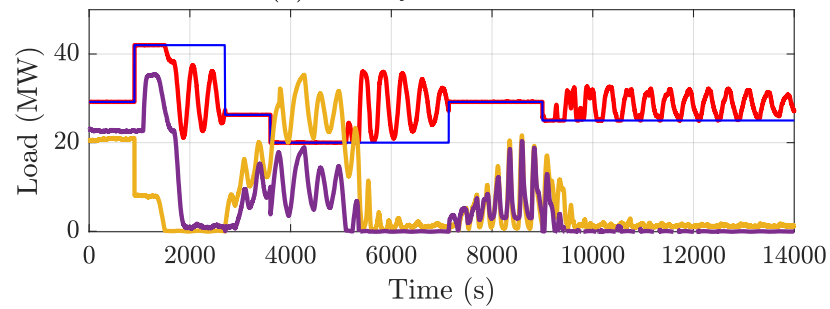

Figure 6: Tracking performance and evolution of the locked ON and OFF loads for (a) a stochastic controller and (b) a priority-stack controller.

which is not apparent when the stochastic controller is used. Finally, it is interesting to note that the stochastic controller dampens the oscillation starting at $t=10000 \mathrm{~s}$, whereas the priority-stack controller is unable to do so.

\section{CONCLUSION}

We have presented a method to handle lockout constraints when describing aggregate TCL flexibility of TCL. The method modifies the lockout durations, such that user-comfort is guaranteed under any external commands without relying on local-controller computations. This allows us to derive analytical expressions for the reduced energy and power flexibility of the thermal battery model. These analytical expressions were verified in simulations and were shown to capture the flexibility reduction very well. Finally, we identified the advantages of a stochastic controller against a priority-stack controller. Besides the fact that the stochastic controller does not require temperature information, it is also more robust against infeasible reference signals. Therefore, it reduces oscillations in the TCL population, which are pronounced with priority-stack controllers. Future work will focus on generalizing the power constraints by tracking the number of locked loads.

\section{ACKNOWLEDGMENTS}

C. Ziras, S. You and H.W. Bindner would like to acknowledge the support of the EUDP project Ecogrid 2.0 (grant 64015 - 0082, website: www.ecogrid.dk).

\section{REFERENCES}

[1] Y. V. Makarov, C. Loutan, J. Ma, and P. de Mello, "Operational impacts of wind generation on California power systems," IEEE Transactions on Power Systems, vol. 24, no. 2, pp. 1039-1050, 2009.

[2] J. L. Mathieu, M. E. H. Dyson, and D. S. Callaway, "Resource and revenue potential of California residential load participation in ancillary services," Energy Policy, vol. 80, pp. 76-87, 2015.

[3] J. L. Mathieu, M. Kamgarpour, J. Lygeros, G. Andersson, and D. S. Callaway, "Arbitraging intraday wholesale energy market prices with aggregations of thermostatic loads," IEEE Transactions on Power Systems, vol. 30, no. 2, pp. 763-772, 2015.

[4] H. Hao, B. M. Sanandaji, K. Poolla, and T. L. Vincent, "A generalized battery model of a collection of Thermostatically Controlled Loads for providing ancillary service," 2013 51st Annual Allerton Conference on Communication, Control, and Computing, Allerton, pp. 551-558, 2013.

[5] — "Aggregate flexibility of thermostatically controlled loads," IEEE Transactions on Power Systems, vol. 30, no. 1, pp. 189-198, 2015.

[6] L. Zhao, W. Zhang, H. Hao, and K. Kalsi, "A geometric approach to aggregate flexibility modeling of thermostatically controlled loads," IEEE Transactions on Power Systems, vol. PP, no. 99, pp. 1-1, 2017.

[7] B. M. Sanandaji, T. L. Vincent, and K. Poolla, "Ramping Rate Flexibility of Residential HVAC Loads," IEEE Transactions on Sustainable Energy, vol. 7, no. 2, pp. 865-874, 2016.

[8] W. Zhang, J. Lian, C. Y. Chang, and K. Kalsi, "Aggregated modeling and control of air conditioning loads for demand response," IEEE Transactions on Power Systems, vol. 28, no. 4, pp. 4655-4664, 2013.

[9] M. Liu and Y. Shi, "Model Predictive Control of Aggregated Heterogeneous Second-Order Thermostatically Controlled Loads for Ancillary Services," IEEE Transactions on Power Systems, vol. 31, no. 3, pp. 1963-1971, 2016.

[10] L. C. Totu, R. Wisniewski, and J. Leth, "Demand Response of a TCL population using Switching-Rate Actuation," IEEE Transactions on Control Systems Technology, pp. 1-16, 2016.

[11] Z. Xu, D. S. Callaway, Z. Hu, and Y. Song, "Hierarchical Coordination of Heterogeneous Flexible Loads," IEEE Transactions on Power Systems, vol. 31, no. 6, pp. 4206-4216, 2016.

[12] C. Ziras, E. Vrettos, and G. Andersson, "Primary frequency control with refrigerators under startup dynamics and lockout constraints," 2015 IEEE Power Energy Society General Meeting, pp. 1-5, July 2015.

[13] E. Vrettos, C. Ziras, and G. Andersson, "Fast and Reliable Primary Frequency Reserves From Refrigerators with Decentralized Stochastic Control,' IEEE Transactions on Power Systems, vol. 32, no. 4, pp. 2924 2941, 2017.

[14] J. T. Hughes, A. D. Domínguez-García, and K. Poolla, "Identification of Virtual Battery Models for Flexible Loads," IEEE Transactions on Power Systems, vol. 31, no. 6, pp. 4660-4669, 2016.

[15] H. Hao, D. Wu, J. Lian, and T. Yang, "Optimal coordination of building loads and energy storage for power grid and end user services," IEEE Transactions on Smart Grid, vol. PP, no. 99, pp. 1-1, 2017.

[16] J. L. Mathieu, M. Dyson, and D. S. Callaway, "Using Residential Electric Loads for Fast Demand Response : The Potential Resource and Revenues , the Costs , and Policy Recommendations," Proceedings of the ACEEE Summer Study on Buildings, pp. 189-203, 2012.

[17] E. C. Kara, M. Bergés, and G. Hug, "Impact of disturbances on modeling of thermostatically controlled loads for demand response," IEEE Transactions on Smart Grid, vol. 6, no. 5, pp. 2560-2568, 2015.

[18] J. L. Mathieu, S. Koch, and D. S. Callaway, "State estimation and control of electric loads to manage real-time energy imbalance," IEEE Transactions on Power Systems, vol. 28, no. 1, pp. 430-440, 2013. 